\title{
Order as an effect of disorder
}

\author{
J. Villain (*), R. Bidaux, J.-P. Carton and R. Conte \\ DPh-G/PSRM, CEN de Saclay, B.P. No 2, 91190 Gif-s/Yvette, France
}

(Reçu le 9 avril 1980, révisé le 3 juillet, accepté le 11 juillet 1980)

\begin{abstract}
Résumé. - On considère un modèle d'Ising frustré généralisé sur un réseau bidimensionnel. Ce modèle est paramagnétique à température nulle mais ferromagnétique pourvu que $0<T<T_{\mathrm{c}}$. On étudie également l'effet de la dilution sur ce système, et l'on montre que l'ordre à longue distance est rétabli dans le modèle dilué sous certaines conditions de concentration, température et interactions qui sont discutées en comparaison avec la percolation usuelle.
\end{abstract}

\begin{abstract}
A generalized frustrated Ising model on a two-dimensional lattice is considered. This model is paramagnetic at zero temperature but ferromagnetic provided $0<T<T_{\mathrm{c}}$. The effect of dilution on this system is also investigated, and long range order is shown to be restored in the dilute model under certain conditions involving concentration, temperature and interactions which are discussed in comparison with usual percolation.
\end{abstract}

1. Introduction. - A few theoretical models are known to have the following unusual property : they exhibit no long range order when the temperature $T$ is strictly zero, whereas they do at low but finite temperature. An important example is the Ising model on the f.c.c. lattice with antiferromagnetic interactions between nearest neighbours. Computer simulations have detected a first order transition in this model [1].

A similar effect can be derived in a generalized version of the domino model invented by André et al. [2], and defined in the next section. This model does exhibit long range order at low temperature and not at $T=0$, as will be seen in section 3 . The effect of random impurities at frozen positions is investigated in the following sections. It will be shown that, in certain cases, they can restore order (e.g. ferromagnetic order). Thus the unexpected effect of both thermal disorder and quenched stoichiometric disorder is, in certain exceptional systems, to restore magnetic order !

2. The domino model. - The domino model is an Ising model on a rectangular lattice with two kinds of ions A, B (Fig. 1) forming alternating chains parallel to $\mathrm{O} v$. There are 3 interactions $J_{\mathrm{AA}}, J_{\mathrm{BB}}$,

(*) Département de Recherche Fondamentale, Laboratoire de diffraction neutronique, CEN, 85X, 38041 Grenoble Cedex, France.

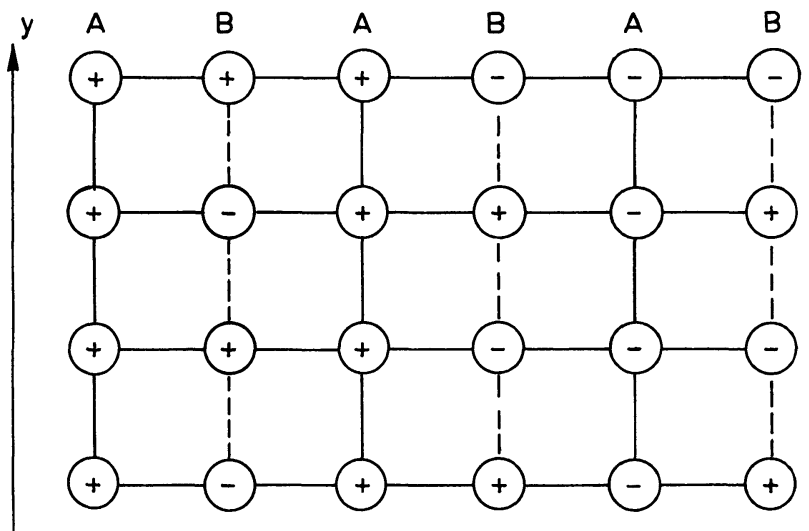

Fig. 1. - The domino model and one of its ground states. Ferromagnetic bonds are full lines, antiferromagnetic bonds are dashed lines.

$J_{\mathrm{AB}}$ between nearest neighbours. A ferromagnetic interaction $J_{\mathrm{AA}}>0$ and an antiferromagnetic interaction $J_{\mathrm{BB}}<0$ will be assumed, so that the model is frustrated [4]. Periodic boundary conditions will be assumed, so that the number $N^{\prime \prime}$ of chains is even as well as the number $N^{\prime}=N / N^{\prime \prime}$ of sites per chain.

André et al. [2] considered a restricted model with $J_{\mathrm{AA}}=J_{\mathrm{AB}}$. The model defined here is more realistic since the Hamiltonian has the symmetry of the problem.

Throughout this paper it will be assumed that

$$
0<J_{\mathrm{AB}}<\left|J_{\mathrm{BB}}\right|<J_{\mathrm{AA}} \text {. }
$$


In this case a ground state corresponds to a ferromagnetic order of each $\mathrm{A}$ chain and an antiferromagnetic order of each B chain (Fig. 1) without any coupling between different chains. The number of such configurations is $g=2^{N^{\prime \prime}}$, nevertheless the residual entropy per spin is zero at $T=0$.

The zero temperature correlation functions are :

$$
\left\langle S_{i} S_{j}\right\rangle=\frac{1}{g} \sum_{\alpha}\left\langle\alpha\left|S_{i} S_{j}\right| \alpha\right\rangle
$$

where $|\alpha\rangle$ denotes the various ground states and $\langle\alpha|X| \alpha\rangle$ is the value of the quantity $X$ in the state $|\alpha\rangle$.

If inequality (2.1) is satisfied, the correlation functions $\left\langle S_{i} S_{j}\right\rangle$ are easily seen to vanish except if $i$ and $j$ are on the same row parallel to $O y$. The average magnetization $m$ per spin vanishes in the thermodynamic limit $(N \rightarrow \infty)$ since :

$$
m^{2}=\frac{1}{N^{2}} \sum_{i j}\left\langle S_{i} S_{j}\right\rangle
$$

where $N$ is the number of spins. Any staggered magnetization also vanishes and the ground state is therefore disordered [9]. The restricted model considered by André et al. turns out to be equivalent, via a Mattis transformation [3, 4] to a model with

$$
J_{\mathrm{AA}}>J_{\mathrm{AB}}=-J_{\mathrm{BB}}>0 \text {. }
$$

In this restricted model the ground state magnetization $m$ defined by (2.3) does not vanish because almost all ground states $|\alpha\rangle$ are ferromagnetic though there are some ground states with zero magnetization.

3. Effect of temperature. - In the previous section, the generalized domino model defined by relations (2.1) was found to be non-magnetic at $T=0$. However, it undergoes a phase transition. Indeed, the exact partition function $Z$ can be calculated via Pfaffian or transfer matrix methods [2] and turns out to be :

$$
\begin{aligned}
\frac{1}{N} \ln Z=\ln 2 & +\frac{1}{16 \pi^{2}} \int_{0}^{2 \pi} \int_{0}^{2 \pi} \ln \left\{\frac { 1 } { 2 } \left[\cosh 4 \beta J_{\mathrm{AA}} \cosh 4 \beta J_{\mathrm{BB}}\right.\right. \\
& +\cosh ^{2} 4 \beta J_{\mathrm{AB}} \cosh 4 \beta\left(J_{\mathrm{AA}}+J_{\mathrm{BB}}\right)-\sinh ^{2} 4 \beta J_{\mathrm{AB}} \cos \varphi \\
& \left.\left.-2 \cosh 4 \beta J_{\mathrm{AB}} \sinh 4 \beta\left(J_{\mathrm{AA}}+J_{\mathrm{BB}}\right) \cos \theta+\sinh 4 \beta J_{\mathrm{AA}} \sinh 4 \beta J_{\mathrm{BB}} \cos 2 \theta\right]\right\} \mathrm{d} \varphi \mathrm{d} \theta
\end{aligned}
$$

where $-2 J_{\mathrm{AA}},-2 J_{\mathrm{BB}}$ and $-2 J_{\mathrm{AB}}$ designate the energy of a pair of parallel interacting spins, so that our notations differ by a factor 2 from those of André et al. [2].

A singularity is found at a temperature $T_{\mathrm{c}}=1 / k_{\mathrm{B}} \beta_{\mathrm{c}}$ defined by :

$$
\sinh 2 \beta_{\mathrm{c}}\left|J_{\mathrm{AA}}+J_{\mathrm{BB}}\right| \sinh 4 \beta_{\mathrm{c}} J_{\mathrm{AB}}=1 .
$$

What is the nature of the state which appears below $T_{\mathrm{c}}$ ? It will be shown further on to be ferromagnetically ordered - or ferrimagnetically ordered, it is just a question of language.

The best way to see that is to eliminate B spins as follows. Let A spins have given values; their contribution to the local field at any site $j$ occupied by a B spin is $4 J_{\mathrm{AB}} \varepsilon_{j}$, where $\varepsilon_{j}=0,1$ or -1 . is :

The partition function of a B chain of length $N^{\prime}$

$$
\begin{aligned}
Z=\sum_{S_{1}, S_{2}, \ldots, S_{N^{\prime}}= \pm 1} \exp \beta \sum_{j=1}^{N^{\prime}}\left[4 J_{\mathbf{A B}} S_{j} \varepsilon_{j}-\right. \\
\left.-2\left|J_{\mathbf{B B}}\right| S_{j} S_{j+1}\right]
\end{aligned}
$$

If the actual calculation of this quantity is possible, elimination of the B spins from the problem leads to the definition of effective interactions between the spins of two A chains sandwiching a B chain by writing

$$
Z=\exp -\beta \mathcal{H}_{\text {eff }} .
$$

One is then left with a system of A spins, for which indirect interactions arising from $\mathscr{H}_{\text {eff }}$ are to be added to the direct interactions $J_{\mathbf{A A}}$.

When conditions (2.1) are satisfied, $Z$ can be calculated at low temperature :

$$
k_{\mathrm{B}} T \ll J_{\mathrm{AB}} .
$$

The detailed calculation is given in Appendix A. A simplified version will be given now, in which each A chain is adequately treated as a saturated ferromagnetic object. If a B chain of length $v$ is sandwiched between two A chains with parallel spins, the lowest excitations of the B chain (Fig. 2a) have an energy $4\left(\left|J_{\mathrm{BB}}\right|-J_{\mathrm{AB}}\right)$ and their number is $v / 2$. The partition function of the $B$ chain is therefore approximately (the energy of the antiferromagnetic ground level defining the zero energy) :

$$
Z_{\mathrm{F}} \simeq\left[1+\exp -4 \beta\left(\left|J_{\mathbf{B B}}\right|-J_{\mathrm{AB}}\right)\right]^{v / 2} .
$$

If the two A chains have opposite spins (Fig. 2b) the elementary excitations of the B chain have an 


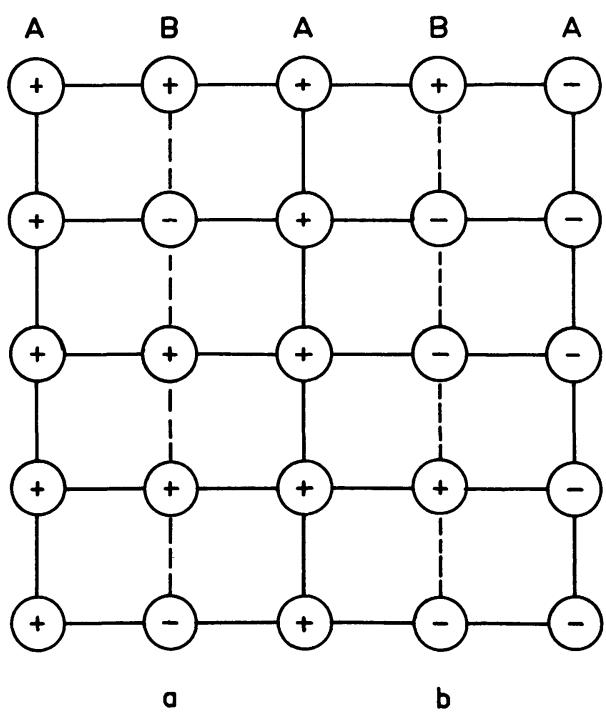

Fig. 2. - Excited state of the domino model. Excitation (a) has lower energy than excitation $(b)$. This produces an effective ferromagnetic coupling between A chains if $J_{\mathbf{A A}}>\left|J_{\mathbf{B B}}\right|$.

energy $4\left|J_{\mathrm{BB}}\right|$ and their number is $v$, which leads to the approximate partition function of the $B$ chain :

Hence

$$
Z_{\mathrm{AF}} \simeq\left[1+\exp -4 \beta\left|J_{\mathrm{BB}}\right|\right]^{v} .
$$

$Z_{\mathrm{F}} / Z_{\mathrm{AF}} \simeq\left[1+\frac{1}{2} \exp -4 \beta\left(\left|J_{\mathrm{BB}}\right|-J_{\mathrm{AB}}\right)\right]^{v}$.

One can notice that the partition function per spin of the infinite Ising chain with antiferromagnetic exchange $2 J_{\mathrm{BB}}$ between neighbouring spins, in the presence of a uniform applied field $H$, is given by

$$
\begin{aligned}
z_{H} & =\exp -2 \beta\left|J_{\mathrm{BB}}\right| \cosh \beta H+ \\
& +\left[\exp -4 \beta\left|J_{\mathrm{BB}}\right| \sinh ^{2} \beta H+\exp 4 \beta\left|J_{\mathrm{BB}}\right|\right]^{1 / 2}
\end{aligned}
$$

and that, to first order in exponential terms at low temperature

$$
\left[\frac{z_{H=4 J_{\mathrm{AB}}}}{z_{0}}\right]^{v}=\frac{Z_{\mathrm{F}}}{Z_{\mathrm{AF}}}
$$

as could be expected.

Taking advantage once more of the low temperature limit, an alternative form of (3.5) is :

$$
\begin{aligned}
Z_{\mathrm{F}} / Z_{\mathrm{AF}} \simeq \exp \left[\frac{v}{2} \exp -4 \beta\left(\left|J_{\mathrm{BB}}\right|-J_{\mathrm{AB}}\right)\right] & = \\
& =\exp 4 \beta J^{\prime} v
\end{aligned}
$$

where

$$
\beta J^{\prime}=\frac{1}{8} \exp -4 \beta\left(\left|J_{\mathbf{B B}}\right|-J_{\mathrm{AB}}\right)
$$

so that the effective Hamiltonian $\mathscr{H}_{\text {eff }}$ defined by (3.4) is seen to be a sum of interactions $-2 J^{\prime} S_{j}^{\mathbf{A}} S_{j}^{\mathbf{A}^{\prime}}$ between facing spins of two neighbouring A columns, which is justified only at low temperature. This result is so simple because $Z_{\mathrm{F}} / Z_{\mathrm{AF}}$ is expressible as a $v$ power.

Thus, eliminating B spins amounts to creating an effective horizontal interaction $J^{\prime}$ between spins located on neighbouring A chains. Another effect, which is derived in Appendix A, is a renormalization of the intra-chain interaction $J_{\text {AA }}$ which should be replaced by $\left(J_{\mathrm{AA}}+\delta J_{\mathrm{AA}}\right)$ with

$$
\delta J_{\mathrm{AA}}=-J_{\mathrm{AB}}+\frac{1}{2 \beta} \ln 2 .
$$

Therefore, the system of A spins reduces at low temperature to a rectangular Ising model with interactions $J^{\prime}$ and $J^{\prime \prime}=J_{\mathrm{AA}}+\delta J_{\mathrm{AA}}$ between nearest neighbours. Its average magnetization $m_{\mathrm{A}}$ per site is given by the standard formula [7] :

$$
\begin{aligned}
& m_{\mathrm{A}}^{8}=1-\frac{1}{\sinh ^{2} 4 \beta J^{\prime} \sinh ^{2} 4 \beta J^{\prime \prime}} \\
& m_{\mathrm{A}} \simeq 1-\frac{1}{8} \exp -8 \beta\left(J_{\mathrm{AA}}-\left|J_{\mathrm{BB}}\right|\right) .
\end{aligned}
$$

Formula (3.9) shows that the A system is ferromagnetic at low temperature $T$, and $m_{\mathrm{A}}$ takes the limit value 1 when $T$ goes to zero. Since $m_{\mathrm{A}}$ is zero at $T=0$ it may be helpful to consider the effect of the stage at which the thermodynamic limit is carried out. What we have proved in this section is :

$$
\lim _{T \rightarrow 0} \lim _{N \rightarrow \infty} m_{\mathrm{A}}=1 .
$$

However it can easily be checked that the limits cannot be interchanged, in contrast with the nonfrustrated case $J_{\mathrm{AA}}, J_{\mathrm{BB}}>0$ :

$$
\lim _{N \rightarrow \infty} \lim _{T \rightarrow 0} m_{\mathrm{A}}=\lim _{N \rightarrow \infty}\left(m_{\mathrm{A}}\right)_{T=0}=0
$$

in agreement with section 2.

As a concluding remark to this section, one may note that the average magnetization $m_{\mathrm{B}}$ per site of a $\mathrm{B}$ chain can be deduced from (3.6): in the low temperature limit each site of the chain undergoes a uniform applied field $4 J_{\mathrm{AB}}$ and one finds :

$$
\begin{aligned}
& m_{\mathrm{B}}=\frac{\sinh 4 \beta J_{\mathrm{AB}}}{\left[\sinh ^{2} 4 \beta J_{\mathrm{AB}}+\exp 8 \beta\left|J_{\mathrm{BB}}\right|\right]^{1 / 2}} \\
& m_{\mathrm{B}} \simeq \frac{1}{2} \exp -4 \beta\left(\left|J_{\mathrm{BB}}\right|-J_{\mathrm{AB}}\right) .
\end{aligned}
$$

Comparison with (3.9) shows that the net magnetization per site $m=\frac{m_{\mathrm{A}}+m_{\mathrm{B}}}{2}$ may have two types of 
behaviour with increasing temperature, according as $\left|J_{\mathrm{BB}}\right|-J_{\mathrm{AB}} \gtrless 2\left(J_{\mathrm{AA}}-\left|J_{\mathrm{BB}}\right|\right)$ (Fig. 3) $\left(^{1}\right)$.

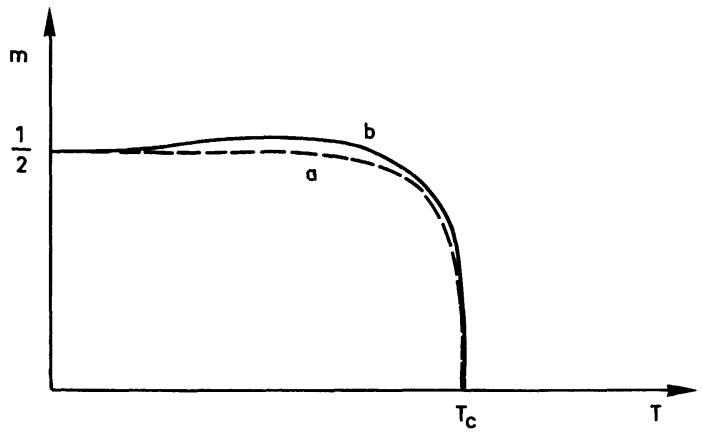

Fig. 3. - Net magnetization curve of the generalized domino model : a) $\left.\quad\left|J_{\mathrm{BB}}\right|-J_{\mathrm{AB}}>2\left(J_{\mathrm{AA}}-\left|J_{\mathrm{BB}}\right|\right) ; \quad b\right)$ $\left|J_{\mathbf{B B}}\right|-J_{\mathbf{A B}}<2\left(J_{\mathbf{A A}}-\left|J_{\mathbf{B B}}\right|\right)$; in the latter case, location and amplitude of the extremum are only descriptive.

4. The impure domino model. - It will now be assumed that given fractions $x_{\mathrm{A}}$ and $x_{\mathrm{B}}$ of sites $\mathrm{A}$ and $\mathrm{B}$ are substituted by non-magnetic impurities. For each distribution of impurities, the system has one or several ground states of energy $W_{\mathrm{GS}}$. Let $W_{0}$ be the sum of the minimum energies $-2\left|J_{i j}\right|$ of all bonds. Because of frustration, $W_{\mathrm{GS}}$ is generally higher than $W_{0}$. The following decomposition will be useful :

$$
W_{\mathrm{GS}}=W_{0}+\sum_{i j} w_{i j}
$$

where

$$
w_{i j}=\left|J_{i j}\right|-J_{i j} S_{i}^{0} S_{j}^{0}
$$

and $\left\{S_{i}^{0}\right\}$ is the set of spin values in one particular ground state. Expression (4.1) can be written as :

$$
W_{\mathrm{GS}}=W_{0}+W_{1}+\sum_{\rho} w_{\rho}
$$

where $\rho$ labels the plaquettes (i.e. rectangles of 4 bonds [4]), and

$$
w_{\rho}=\sum_{i<j} 2 w_{j} \mu_{i j}
$$

where the sum is over the 4 bonds of the plaquette $\rho$ and $\mu_{i j}=1 / 2$ for a bond (ij) which belongs to two plaquettes, otherwise $\mu_{i j}=1$.

$W_{1}$ is the sum of the contributions $2 w_{i j}$ of bonds which do not belong to any plaquette. It results from (4.2) and (4.4) that

$$
W_{1} \geqslant 0, \quad w_{\rho}>0 .
$$

(1) The Maxwell relation $(\partial m / \partial T)_{H}=(\partial S / \partial H)_{T}$ shows that the unusual positive slope found at low temperature for $m(T)$ in case b) is associated with the fact that the system will increase its entropy in presence of a small uniform applied field, which is reminiscent of the behaviour of the antiferromagnetic Ising chain.
No complete study of the situation at $T=0$ will be given but somewhat unexpected qualitative results will be derived in certain special cases.

5. An exactly solvable case. - The ground states can be determined exactly when $x_{\mathrm{A}}=0$ and

$$
2 J_{\mathrm{AB}}<\left|J_{\mathrm{BB}}\right|<J_{\mathrm{AA}} .
$$

Condition (5.1) implies, as seen below, that in the ground states all A-pairs are parallel and all B-pairs are antiparallel. With the less stringent condition (2.1) the characterization of the ground state is a little more tricky.

A sites form infinite chains and B sites form finite chains as shown by figure 4 . Let a fragment of B chain

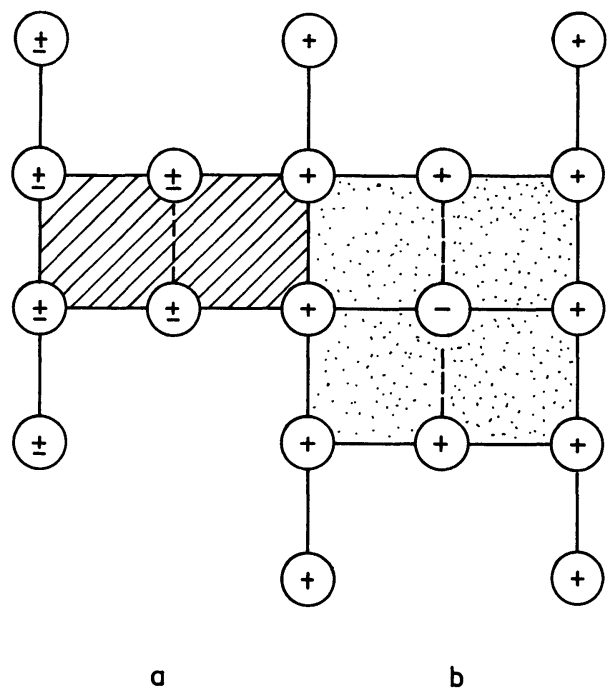

Fig. 4. - Ground states of the impure domino model. Even B chains ( $a$ ) do not couple A chains. Odd B chains $(b)$ couple them ferromagnetically. Dashed and dotted areas represent two $\Gamma$ sets.

have $v$ spins; its bonds belong to a set $\Gamma$ of $2(v-1)$ plaquettes. Equation (4.3) can be written as

$$
W_{\mathrm{GS}}=W_{0}+W_{1}+\sum_{\Gamma} w(\Gamma)
$$

where

$$
w(\Gamma)=\sum_{\rho \in \Gamma} w_{\rho}
$$

Each term of (5.2) can be minimized separately by the same states, which are therefore ground states. This is shown below.

i) Case of sets $\Gamma$ with an even number $v$ of spins. $w(\Gamma)$ is minimum if the following conditions are fulfilled : $a$ ) the B spins point alternately up and down. b) The A spins on the right hand side have the same sign $\sigma . c$ ) The A spins on the left hand side have the same sign $\sigma^{\prime}(= \pm \sigma)$ (Fig. $4 a$ ). 
ii) Case of sets $\Gamma$ with an odd number $v$ of spins. $w(\Gamma)$ is minimum if the following conditions are fulfilled : a) All A spins have the same sign $\sigma= \pm$. $b$ ) The B spins are alternately up and down. $c$ ) Both end spins of type B have sign $\sigma$ (Fig. $4 b$ ).

iii) $W_{1}$ vanishes, and is minimum according to (4.5), if all bonds which do not belong to any plaquette have their spins parallel.

All conditions (i), (ii) and (iii) are compatible. Taken together, they generally imply that all A spins have the same sign. The ground state is therefore ferromagnetic (or ferrimagnetic, it is a question of language). Only odd B chains contribute to the ferromagnetic coupling.

Each odd B chain has a well-defined state with magnetization +1 if $A$ spins are up ( -1 otherwise). Each even B chain has two possible states and no magnetization. Thus, if there are $n^{\prime}$ odd B chains, $n^{\prime \prime}$ even B chains, and $\frac{N}{2} \mathrm{~A}$ spins, the system has $2^{n^{\prime \prime}}$ ground states which all have the same total magnetization $M=\frac{N}{2}+n^{\prime}$.

We have reached the following, surprising conclusion. In the frustrated domino model, if condition (5.1) is satisfied, non-magnetic impurities on B sites generally produce ferromagnetic order though the ground state degeneracy is generally increased. The word generally is a politeness to Mathematicians since $n^{\prime}$ or (and) $n^{\prime \prime}$ can in principle vanish.

6. The case $x_{\mathrm{A}} \ll x_{\mathrm{B}}$. - When $x_{\mathrm{A}}$ does not strictly vanish but is much smaller than $x_{\mathrm{B}}$, an approximate treatment can be given if condition (5.1) is satisfied.

For any given value of $x_{\mathrm{A}}$, there is a percolation threshold $X\left(x_{\mathrm{A}}\right)$ such that for $x_{\mathrm{B}}>X\left(x_{\mathrm{A}}\right)$ the system is constituted by finite, unconnected clusters.

When $x_{\mathrm{B}}$ is sufficiently smaller than $X\left(x_{\mathrm{A}}\right)$ ferromagnetic order is expected to persist. A sites form long ferromagnetic chains which are ferromagnetically coupled together via odd B chains as explained in section 5. Antiferromagnetic coupling is only possible at the ends of A chains (Fig. 5) and this effect can be neglected because very few points are the ends of A chains.

7. Vicinity of the percolation threshold. - In order to make the proofs easier, $x_{\mathrm{A}}$ will again be assumed to be small, although the results are probably general; in this case, $X\left(x_{\mathrm{A}}\right)$ is close to 1 .

When $x_{\mathrm{B}}$ is just a little smaller than the percolation threshold $X\left(x_{\mathrm{A}}\right)$, the system consists of long A chains linked together by a comparatively small number

$$
\frac{N}{2}\left(1-x_{\mathrm{B}}\right) \approx \frac{N}{2}(1-X) \text { of B atoms . }
$$

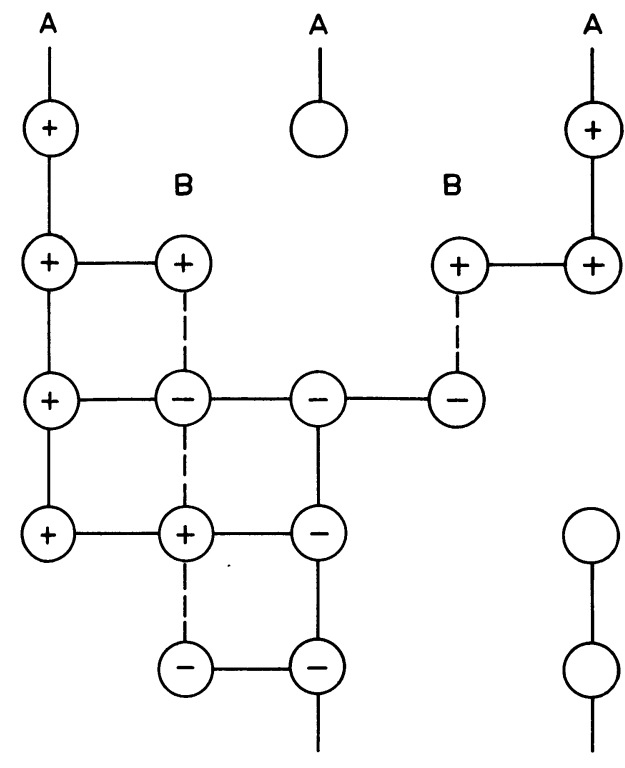

Fig. 5. - Two examples of indirect antiferromagnetic coupling between A chains through B spins.

Most of these atoms, i.e.

$$
\frac{N}{2} x_{\mathbf{B}}^{2}\left(1-x_{\mathbf{B}}\right) \approx \frac{N}{2} X^{2}(1-X),
$$

are isolated; the remainder is distributed among various linear clusters, the number of clusters of size $p$ being

$$
\frac{N}{2} x_{\mathbf{B}}^{2}\left(1-x_{\mathbf{B}}\right)^{p} \approx \frac{N}{2} X^{2}(1-X)^{p}
$$

As seen in section 5, clusters with an even number of B atoms, and therefore pairs, do not contribute to the coupling of A chains; to second order of approximation in $(1-X)$, clusters containing more than two B atoms can be neglected. The concentration of active $B$ atoms therefore reduces to that of the isolated ones. Thus the condition for a ferromagnetic ground state is more stringent than in the non-frustrated case. This condition can of course be written

$$
x_{\mathrm{B}}<X^{\prime}\left(x_{\mathrm{A}}\right)
$$

where $X^{\prime}\left(x_{\mathrm{A}}\right)$ is smaller than the percolation threshold $X\left(x_{\mathrm{A}}\right) \cdot X^{\prime}$ is evaluated in Appendix B where the effect of larger B clusters is examined, but can be guessed by the following argument. Percolation phenomenon is ruled, at $T=0$, by the number of bridges which are able to propagate information between $\mathrm{A}$ chains. In the frustrated domino model, the effective number of these bridges is that of the $\frac{N}{2} x_{\mathbf{B}}^{2}\left(1-x_{\mathbf{B}}\right)$ isolated B spins. In ordinary percolation, any cluster provides one bridge so that the corresponding total number of bridges is $\frac{N}{2} X(1-X)$. The critical concentration $X^{\prime}$ 
in the frustrated domino model is obtained by equating the respective numbers of bridges :

$$
\left[x_{\mathrm{B}}^{2}\left(1-x_{\mathrm{B}}\right)\right]_{x_{\mathrm{B}}=X^{\prime}}=X(1-X),
$$

so that, to second order in $1-X$ :

$$
X^{\prime}=X-(1-X)^{2} \text {. }
$$

A sufficient condition for the system to be paramagnetic at all temperatures is derived in Appendix C :

$$
x_{\mathrm{B}}>X-\left(\frac{1-X}{2}\right)^{2}=X^{\prime \prime}\left(x_{\mathrm{A}}\right) \text {. }
$$

We have not been able to determine what occurs in the region $X^{\prime}<x_{B}<X^{\prime \prime}$. The possibility of two phase transitions is not excluded. This phenomenon does occur in certain models as shown in the next section.

The result derived for the impure domino model at zero temperature are collected in figure 6 .

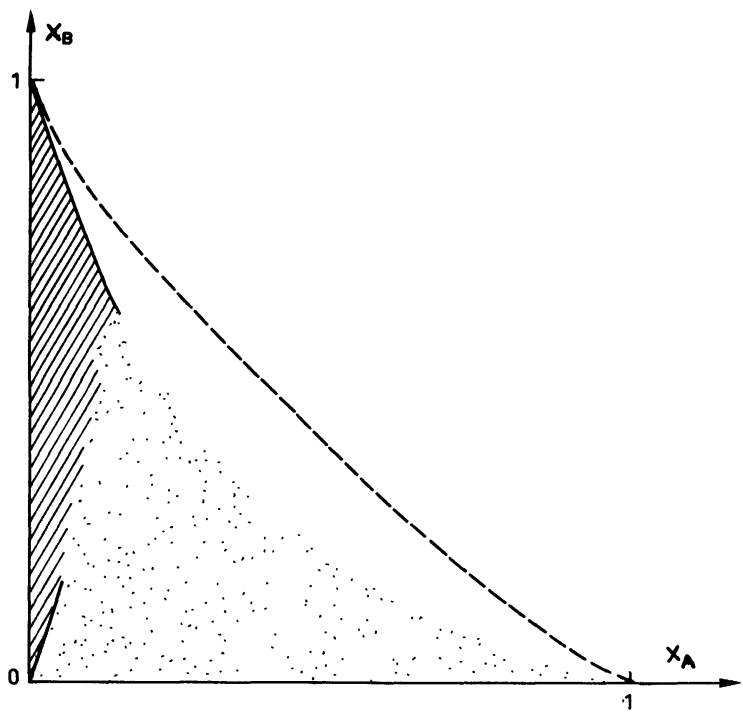

Fig. 6. - Phase diagram of the impure domino model ground state when $J_{\mathrm{AA}}>-J_{\mathbf{B B}}>2 J_{\mathrm{AB}}$. White area : paramagnetic region. Dashed area : ferromagnetic region. Dotted area : not investigated in this work. Point $\mathbf{0}$ is paramagnetic.

8. A model with two phase transitions at finite temperature. - The model which will be studied in this section is a rather artificial one. Its interest is to exhibit another spectacular effect of frustration in presence of non-magnetic impurities : it is disordered at low and high temperatures, and ferromagnetic in some intermediate range. This property is known to occur in certain solids : Rochelle salt, terbium gadolinium vanadate, barium sodium niobate [6]. Certain solutions like water-nicotine exhibit similar features. Although the model presented here has little kinship with the productions of nature, it is simple and preserves most of the conditions leading to the situation analysed.

The main effect of inserting non-magnetic impurities into the $B$ lattice, i.e. the creation of clusters with an even number of $B$ atoms, is enhanced by considering a decorated model where all B sites are paired; the unit cell of this model is displayed in figure 7. Now B spins can easily be eliminated from the problem and one is left with A spins coupled by an effective horizontal interaction $J^{\prime}$ between nearest neighbours defined by

$$
4 \beta J^{\prime}=\ln \frac{1+\mathrm{e}^{-4 \beta\left|J_{B B}\right|} \cosh 8 \beta J_{\mathrm{AB}}}{1+\mathrm{e}^{-4 \beta\left|J_{B B}\right|}}
$$

or at low $T$ if (5.1) holds :

$$
J^{\prime} \approx \frac{1}{8} k_{\mathrm{B}} T \exp -4 \beta\left(\left|J_{\mathrm{BB}}\right|-2 J_{\mathrm{AB}}\right) .
$$

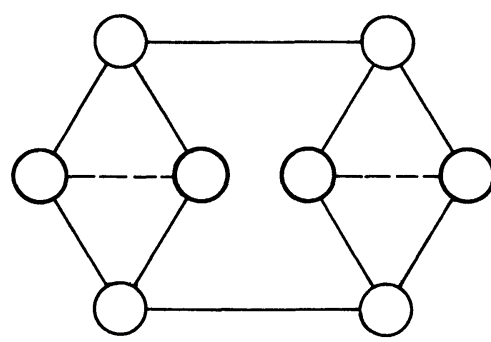

Fig. 7. - Unit cell of the decorated model considered in section 8 .

In absence of impurities the system is ordered at low temperature and paramagnetic at $T=0$, like the domino model. A more interesting situation arises when non-magnetic impurities are inserted into the A lattice with concentration $x$. At low temperature there is complete order inside each piece of A chain, and the effective horizontal interaction between two adjacent pieces is about $J^{\prime} / x$, which should be larger than $k_{\mathrm{B}} T$ to produce any order. Therefore a lower critical temperature $T_{\mathrm{c}}^{-}$appears, the order of magnitude of which being [5]

$$
k_{\mathrm{B}} T_{\mathrm{c}}^{-} \sim \frac{J^{\prime}\left(T_{\mathrm{c}}^{-}\right)}{x}
$$

or, for small values of $x$

$$
k_{\mathrm{B}} T_{\mathrm{c}}^{-} \sim \frac{4\left(2 J_{\mathrm{AB}}-\left|J_{\mathrm{BB}}\right|\right)}{\ln 8 x} .
$$

The higher critical temperature does not depend crucially on $x$ and may be approximated by the transition condition for $x=0$ :

$$
\sinh 4 \beta_{\mathrm{c}}^{+} J_{\mathrm{AA}} \sinh 4 \beta_{\mathrm{c}}^{+} J^{\prime} \approx 1
$$

where $J^{\prime}$ is given by $(8.1)$. The system is ferromagnetic 
for $T_{\mathrm{c}}^{-}<T<T_{\mathrm{c}}^{+}$, and paramagnetic outside this region.

It is worth noticing that insertion of impurities on B sites as well would restore ferromagnetism at $T=0$.

9. Conclusion. - The pure generalized domino model exhibits ferromagnetic long range order at low temperature but not at $T=0$. This is very reminiscent of the effect observed in the f.c.c. Ising antiferromagnet, and in so far as the role of frustration prevails on that of dimensionality it is pleasant to deal with a model which is tractable by analytical methods. At $T=0$ the system can become ferromagnetic when non-magnetic impurities are inserted. The phase diagram (Fig. 6) shows large unexplored areas. It would be interesting to know whether the system exhibits some spin-glass characters in certain regions.

The antiferromagnetic case $\left(-J_{\mathbf{B B}}>J_{\mathrm{AA}}>\left|J_{\mathrm{AB}}\right|\right)$ can be transformed into the ferromagnetic case studied above if a Mattis transformation [3, 4] is performed.

The effect of non-magnetic impurities in Heisenberg magnets without long range order in the ground state has been discussed in reference [8]. It was argued that certain systems may become spin glasses; in other cases magnetic order could be restored. The interest of the present example is that the effects of both thermal disorder and stoichiometric disorder can be investigated, and are found to be similar.

The domino model is not unrealistic, and hopefully the corresponding physical realizations may be available some day, and it would be interesting to check the present predictions experimentally.

\section{APPENDIX A}

Derivation of formulae (3.7) and (3.8). - The expression (3.3) can be evaluated by the usual transfer matrix method :

$$
Z=\operatorname{Tr}\left(\hat{\theta}_{1} \hat{\theta}_{2} \hat{\theta}_{3} \ldots \hat{\theta}_{N^{\prime}}\right)
$$

where $\hat{\theta}_{j}$ is a $2 \times 2$ matrix, the lines and columns of which can be denoted by the indices $S, S^{\prime}= \pm 1$. Its matrix elements are :

$$
\begin{aligned}
\left\langle S\left|\hat{\theta}_{j}\right| S^{\prime}\right\rangle=\exp -\{2 \beta \times \\
\left.\times\left[\left|J_{\mathrm{BB}}\right| S S^{\prime}-J_{\mathrm{AB}}\left(\varepsilon_{j} S+\varepsilon_{j+1} S^{\prime}\right)\right]\right\} .
\end{aligned}
$$

In absence of translational invariance the matrices $\hat{\theta}_{j}$ are not all identical. For a given set $\left\{\varepsilon_{j}\right\}$ the B chain can be divided in a certain number $2 M$ of domains of lengths $r_{1}, r_{2}, \ldots, r_{m}, \ldots, r_{2 M}$ inside which the molecular field $4 J_{\mathrm{AB}} \varepsilon_{j}=4 J_{\mathrm{AB}} \varepsilon_{m}$ is uniform. Let $\varepsilon_{m}$ be zero for even values of $m$, and $\varepsilon_{m}= \pm 1$ for odd values. The possibility for $\varepsilon_{m}$ to change abruptly from -1 to +1 may be discarded at low temperature. The lengths $r_{m}$ are defined in such a way that $\left(r_{1}+r_{2}+\cdots+r_{2 M}\right)$ is the total chain length $N^{\prime}$. Expression (A.1) can be written as follows :

$$
\begin{aligned}
Z=\operatorname{Tr} \hat{\theta}_{\varepsilon_{1}}^{r_{1}-1} \hat{U}_{\varepsilon_{1}} \hat{\theta}_{0}^{r_{2}-1} & \hat{U}_{\varepsilon_{3}}^{*} \hat{\theta}_{\varepsilon_{3}}^{r_{3}-1} \hat{U}_{\varepsilon_{3}} \times \\
& \times \hat{\theta}_{0}^{r_{4}-1} \ldots \hat{\theta}_{0}^{r_{2 M}-1} \hat{U}_{\varepsilon_{1}}^{*}
\end{aligned}
$$

where the transfer matrices $\hat{\theta}_{ \pm}, \hat{\theta}_{0}$ and $\hat{U}_{ \pm}$have the following matrix elements deduced from (A.2) :

$$
\begin{aligned}
\left\langle S\left|\hat{\theta}_{ \pm}\right| S^{\prime}\right\rangle & =\exp -2 \beta\left[\left|J_{\mathrm{BB}}\right| S S^{\prime} \mp J_{\mathrm{AB}}\left(S+S^{\prime}\right)\right] \\
\left\langle S\left|\hat{\theta}_{0}\right| S^{\prime}\right\rangle & =\exp -2 \beta\left|J_{\mathrm{BB}}\right| S S^{\prime} \\
\left\langle S\left|\hat{U}_{ \pm}\right| S^{\prime}\right\rangle & =\left\langle S^{\prime}\left|\hat{U}_{ \pm}^{*}\right| S\right\rangle=\exp -2 \beta\left[\left|J_{\mathrm{BB}}\right| S S^{\prime} \mp J_{\mathrm{AB}} S\right] .
\end{aligned}
$$

At low temperature most of the lengths $r_{m}$ are very large and the effect of the lowest eigenvalues of the matrices $\hat{\theta}_{0}$ and $\hat{\theta}_{ \pm}$can be neglected. The following approximations can be used :

$$
\begin{aligned}
& \hat{\theta}_{0} \simeq \mathrm{e}^{2 \beta\left|J_{\mathbf{B B}}\right|} \theta_{0}\left|\xi_{0}\right\rangle\left\langle\xi_{0}\right| \\
& \hat{\theta}_{ \pm} \simeq \mathrm{e}^{2 \beta\left|J_{\mathbf{B B}}\right|} \theta_{1}\left|\xi_{ \pm}\right\rangle\left\langle\xi_{ \pm}\right|
\end{aligned}
$$

where $\left|\xi_{\varepsilon}\right\rangle$ is the eigenvector of $\hat{\theta}_{\varepsilon}$ which corresponds to the largest eigenvalue $\left(\theta_{\varepsilon} \exp 2 \beta\left|J_{\mathbf{B B}}\right|\right)$. Insertion of (A.5) into (A.3) yields :

$$
\begin{aligned}
Z=\mathrm{e}^{2 \beta N^{\prime}\left|J_{B B}\right|} \theta_{1}^{N^{\prime}-2 M}\left(\theta_{0} / \theta_{1}\right)^{r_{2}+r_{4}+r_{6}+\ldots+r_{2 M}-M} \times \\
\times \prod_{m=1}^{M}\left|\left\langle\xi_{\varepsilon_{m}}\left|\hat{U}_{\varepsilon_{m}}\right| \xi_{0}\right\rangle\right|^{2} .
\end{aligned}
$$

The calculation of all factors is straightforward since it reduces to the diagonalization of the $2 \times 2$ matrices defined by (A.4a) and (A.4b). At low temperature one finds :

$$
\begin{array}{r}
\theta_{1} \simeq 1+\exp -4 \beta\left|J_{\mathbf{B B}}\right| \cosh 4 \beta J_{\mathbf{A B}} \\
\theta_{0} / \theta_{1} \simeq 1-\frac{1}{2} \exp -4 \beta\left(\left|J_{\mathbf{B B}}\right|-J_{\mathrm{AB}}\right) \\
\left\langle\xi_{ \pm}\left|\hat{U}_{ \pm}\right| \xi_{0}\right\rangle \simeq\left(1+\exp -4 \beta\left|J_{\mathrm{BB}}\right|\right) \times \\
\times \cosh 2 \beta J_{\mathrm{AB}} .
\end{array}
$$

Inserting these expressions into (A.6) and introducing the notations (3.7) and (3.8), the final result may be written as follows :

$$
\begin{aligned}
Z= & \left(\theta_{1} \exp 2 \beta\left|J_{\mathrm{BB}}\right|\right)^{N^{\prime}}\left(\cosh 2 \beta J_{\mathrm{AB}}\right)^{2 M} \times \\
& \times\left[1-\frac{1}{2} \exp -4 \beta\left(\left|J_{\mathrm{BB}}\right|-J_{\mathrm{AB}}\right)\right]^{v_{2}+v_{4}+\ldots+v_{2 M}+M}
\end{aligned}
$$


The $M$-dependent part of this expression must be identified with the Boltzmann factor corresponding to the excited state, defined by the set $\left\{\varepsilon_{i}\right\}$, of the two A chains sandwiching the $B$ chain whose spins have been eliminated. These chains have their respective spins parallel within a domain of length $r_{1}$, then antiparallel within a domain of length $r_{2}$, etc... The interchain energy of a pair of adjacent spins is $4 J^{\prime}$ if these spins are antiparallel, zero otherwise. The intrachain energy of a pair of adjacent spins is $4 \delta J_{\mathrm{AA}}$ if these spins are antiparallel, zero otherwise, but attention must be paid to the fact that elimination of two adjacent chains of B spins provides a twofold contribution to the effective interactions inside the intermediate A chain. Therefore one can write (A.7) in the following form :

$$
\begin{aligned}
Z= & \left(\theta_{1} \exp 2 \beta\left|J_{\mathrm{BB}}\right|\right)^{N^{\prime}} \exp \left\{-4 \beta J^{\prime} \times\right. \\
& \left.\times\left(r_{2}+r_{4}+\cdots+r_{2 M}\right)\right\} \times \exp -4 M \beta \delta J_{\mathrm{AA}}
\end{aligned}
$$

with $J^{\prime}$ and $\delta J_{\mathrm{AA}}$ given by (3.7) and (3.8), respectively. Of course $\delta J_{\mathrm{AA}}$ is only a renormalized contribution to the intrachain interaction, so that $J_{\mathrm{AA}}+\delta J_{\mathrm{AA}}$ and $J^{\prime}$ are the effective intrachain and interchain interactions for the system of A spins at low temperature.

\section{APPENDIX B}

Derivation of formula (7.1). - For a B chain of length $N^{\prime}$ with a concentration $x_{\mathbf{B}}=x$ of impurities the mean number of isolated spins is $N^{\prime} x^{2}(1-x)$; the mean number of isolated pairs is $N^{\prime} x^{2}(1-x)^{2}$, and more generally the mean number of $p$-clusters (a $p$-cluster is a set of $p$ adjacent B spins delimited by impurities) is $\bar{n}_{p}=N^{\prime} x^{2}(1-x)^{p}$. It can be checked easily that the mean number of clusters is

$$
\bar{n}_{\mathrm{c}}=\sum_{p=1}^{N^{\prime} \rightarrow \infty} n_{p}=N^{\prime} x(1-x)
$$

while

$$
\sum_{p=1}^{N^{\prime} \rightarrow \infty} p n_{p}=N^{\prime}(1-x) \text { as expected }
$$

Let $\mathcal{A}$ and $\mathcal{A}^{\prime}$ be two neighbouring $\mathrm{A}$ chains, and $r$ be the number of $B$ sites which are common neighbours to $\mathcal{A}$ and $\mathcal{A}^{\prime}$ (for instance $r=2$ for the two $A$ chains at the left hand side of Fig. 5). If the system were ferromagnetic, the probability for the two A chains not to be coupled would be equal to the probability $x^{r}$ that the $r$ B sites are occupied by impurities. For the domino model satisfying condition (5.1), it will be proved now that the probability $p_{r}(x)$ that the two A chains are not coupled in the ground state is $y^{r}$ for large $r$, with

$$
y=x+(1-x)^{2}
$$

to second order in $(1-x)$. Formula (7.1) follows immediately.

What has to be expressed is that, in the domino problem, two A chains are uncoupled not only when the number of B spins present on the $r$ sites is zero, but also when $B$ spins are present and every cluster of $B$ spins contains an even number of elements. Therefore we can write :

$$
p_{r}(x)=p_{0}+p_{2}+p_{4}+\cdots+p_{2 k}+\cdots+p_{2[r / 2]}
$$

( $[r / 2]$ is the largest integer $\leqslant r / 2)$,

where $p_{2 k}$ is the probability that exactly $2 k$ B spins are present on the $r$ sites, and are distributed exclusively in clusters of even size. The number of distinguishable configurations corresponding to this event is $\left(\begin{array}{c}r-k \\ k\end{array}\right)$, so that

$$
p_{2 k}=\left(\begin{array}{c}
r-k \\
k
\end{array}\right) x^{r-2 k}(1-x)^{2 k} .
$$

After insertion of this result into (B.2), inspection of $p_{r}(x)$ shows that $q_{r}(x)=x^{-r} p_{r}(x)$ is defined recursively by

$$
q_{r+2}(x)=q_{r+1}(x)+\left(\frac{1-x}{x}\right)^{2} q_{r}(x)
$$

so that, finally

$$
p_{r}(x)=\frac{x^{r}}{C(x)}\left[\left(\frac{1+C(x)}{2}\right)^{r+1}-\left(\frac{1-C(x)}{2}\right)^{r+1}\right]
$$

where $\quad C(x)=\left[1+4\left(\frac{1-x}{x}\right)^{2}\right]^{1 / 2}$.

Expansion of $p_{r}(x)$ yields

$$
p_{r}(x) \approx x^{r}\left[1+\left(\frac{1-x}{x}\right)^{2}\right]^{r+1} \approx y^{r}
$$

where $y$ is defined by (B.1).

\section{APPENDIX C}

Vicinity of the percolation threshold at finite temperature. - We first state the following lemma :

Lemma : Consider adjacent B spins, submitted to a uniform applied field $4 J_{\mathrm{AB}}$. Let $t$ be the probability for an isolated pair of $B$ spins to be antiparallel, namely $t=\left(1+\mathrm{e}^{-4 \beta\left|J_{\mathbf{B B}}\right|} \cosh 8 \beta J_{\mathrm{AB}}\right)^{-1}$, and $t_{2 q}$ the probability for a cluster of $2 q \mathrm{~B}$ spins to form an antiferromagnetic array; then

$$
t_{2 q} \geqslant t^{2 q-1} \text {. }
$$


The proof of the lemma is rather simple and will only be sketched below. A transfer method for even numbers of spins is used so that $\left(Z_{2 q}^{\dagger}, Z_{2 q}^{\downarrow}\right)$ is linearly expressed from $\left(Z_{2 q-2}^{\dagger}, Z_{2 q-2}^{\dagger}\right), Z_{2 q}^{\sigma}$ being the partition function of a set of $2 q$ spins, the last one being $\sigma$. Going to new variables $Z_{2 q}^{\uparrow}+Z_{2 q}^{\downarrow}$ and $Z_{2 q}^{\uparrow}-Z_{2 q}^{\downarrow}$, it readily follows that

$$
\begin{aligned}
Z_{2 q}= & Z_{2 q}^{\dagger}+Z_{2 q}^{\downarrow} \leqslant\left[\left(1+\mathrm{e}^{4 \beta\left|J_{\mathrm{BB}}\right|}\right)+\right. \\
& \left.+\cosh 8 \beta J_{\mathrm{AB}}\left(1+\mathrm{e}^{-4 \beta \mid J_{\mathrm{BB}}}\right)\right] Z_{2 q-2} .
\end{aligned}
$$

From the definition

$$
t_{2 q}=\frac{2 \exp \left[2 \beta(2 q-1)\left|J_{\mathrm{BB}}\right|\right]}{Z_{2 q}}
$$

and inequality (C.2), one shows after some elementary algebra that

$$
t_{2 q} \geqslant t^{2} t_{2 q-2}
$$

so that (C.1) holds, provided condition (5.1) is satisfied.

Notice that (C.1) holds a fortiori if the A spins of the two chains which border the $B$ spins are no longer parallel, since the probability of an antiferromagnetic state for the $2 q$ B spins is minimal when the field contribution to the Boltzmann factor of this state is minimal, i.e. when all the A spins are parallel.

Consider now the average correlation function of 2 given A spins $i$ and $j$, at finite temperature $T=1 / k_{\mathrm{B}} \beta$, which can formally be written

$$
\begin{aligned}
\overline{\left\langle S_{i} S_{j}\right\rangle}=G\left(r_{i}, r_{j}\right) & =\sum_{\Omega} p(\Omega) \sum_{\mathcal{A}, \mathfrak{B}} \rho(\mathcal{A}, \mathfrak{B} ; \Omega, T) \times \\
& \times n_{i}(\Omega) S_{i}(\mathcal{A}) n_{j}(\Omega) S_{j}(\mathcal{A}) \quad(\mathrm{C} .
\end{aligned}
$$

where $\Omega$ denotes the various impurity configurations, $p(\Omega)$ their probabilities, $\mathcal{A}$ and $\mathcal{B}$ the sets of values of $\mathrm{A}$ and B spins respectively, $\rho(\mathcal{A}, \mathcal{B} ; \Omega, T)$ the thermal Gibbs probability of the state $(\mathcal{A}, \mathcal{B})$ at temperature $T$ for a given configuration $\Omega$, and $n_{i}(\Omega)=0$ or 1 , the spin occupation number of site $i$. A set $(\Omega, \mathfrak{B})$ will be called unefficient if

$$
\begin{gathered}
\exists \mathfrak{B}^{\prime}, \forall \mathcal{A}, \exists \mathcal{A}^{\prime}: S_{i}\left(\mathcal{A}^{\prime}\right) S_{j}\left(\mathcal{A}^{\prime}\right)=-S_{i}(\mathfrak{A}) S_{j}(\mathfrak{A}) \\
\text { and } \quad \rho\left(\mathcal{A}^{\prime}, \mathfrak{B}^{\prime} ; \Omega, T\right)=\rho(\mathfrak{A}, \mathfrak{B} ; \Omega, T)
\end{gathered}
$$

in such a way that the correspondence $\mathcal{B}^{\prime} \leftrightarrow \mathcal{B}^{\prime}$, $\mathcal{A} \leftrightarrow \mathcal{A}^{\prime}$ is one to one. Then, the contributions to $G\left(r_{i}, r_{j}\right)$ in (C.3) of unefficient $(\Omega, \mathcal{B})$ cancel by pairs. A sufficient condition for $(\Omega, \mathcal{B})$ to be unefficient is that every path from $i$ to $j$ either is cut by a nonmagnetic impurity or crosses an antiferromagnetically aligned even B cluster : such a path will be called uneffective. So it turns out that

$$
\begin{array}{ll}
G\left(r_{i}, r_{j}\right)=\sum_{\{\Omega, \mathcal{B}\}} p(\Omega) \sum_{\mathcal{A}} \rho(\mathcal{A}, \mathcal{B} ; \Omega, T) & n_{i}(\Omega) S_{i}(\mathcal{A}) \times \\
& \times n_{j}(\Omega) S_{j}(\mathcal{A}) \\
G\left(r_{i}, r_{j}\right) \leqslant \sum_{\{\Omega, \mathcal{S}\}} p(\Omega) \sum_{\mathcal{A}} \rho(\mathcal{A}, \mathcal{B} ; \Omega, T) . &
\end{array}
$$

In these formulas $\sum_{\{\Omega, \$\}}$ stands for a summation over efficient $(\Omega, \Re)$ only.

$$
G\left(r_{i}, r_{j}\right) \leqslant \sum_{\Omega, \mathscr{B}} p(\Omega) \tilde{\rho}(\mathcal{B}, \Omega, T) \eta_{i j}(\Omega, \mathscr{B})
$$

where

$$
\eta_{i j}(\Omega, \mathcal{B})=\left\{\begin{array}{l}
1 \text { if there is at least one effective path } \\
\text { linking } i \text { to } j . \\
0 \text { otherwise. }
\end{array}\right.
$$

and $\tilde{\rho}(\mathfrak{B} ; \Omega, T)=\sum_{\mathcal{A}} \rho(\mathcal{A}, \mathcal{B} ; \Omega, T)$ is the restricted probability for $\mathfrak{B}$.

$$
\text { Thus } \quad\left|\overline{\left\langle S_{i} S_{j}\right\rangle}\right| \leqslant \Pi_{i j}
$$

where $\Pi_{i j}$ if the probability that $i$ and $j$ are linked by some effective path. In standard percolation theory the probability that two A chains having $r$ common neighbouring $B$ sites are directly linked is $1-x_{\mathbf{B}}^{r}$, and the long distance limit of $\Pi_{i j}$ is

$$
\lim _{\boldsymbol{R}_{i j} \rightarrow \infty} \Pi_{i j}\left\{\begin{array}{l}
=0 \text { if } x_{\mathbf{B}} \geqslant X\left(x_{\mathbf{A}}\right) \\
>0 \text { if } x_{\mathbf{B}}<X\left(x_{\mathbf{A}}\right) .
\end{array}\right.
$$

What we need in the present problem is the probability $w_{r}(x)$ that two A chains having $r$ common neighbouring $B$ sites are directly linked, for a given concentration $x_{\mathbf{B}}=x$ of impurities in the B lattice. We shall now prove that, for large $r$,

$$
w_{r}(x)<1-y^{\prime r}
$$

where $y^{\prime}$ depends on $x$ and $T$. Then $y^{\prime} \geqslant X\left(x_{\mathrm{A}}\right)$ will be a sufficient condition for paramagnetism at temperature $T$.

The line we follow now is similar to that used in Appendix B. A sufficient condition for two A chains (having $r$ common neighbouring B sites) not to be linked occurs when an even number of $\mathbf{B}$ spins are present and these spins form clusters of even size, each cluster being antiferromagnetic. Therefore if $p_{r}^{\prime}(x)$ is the probability of this event, one can write

\section{Now}

$$
w_{r}(x)<1-p_{r}^{\prime}(x) .
$$

$$
p_{r}^{\prime}(x)=p_{0}^{\prime}+p_{2}^{\prime}+\cdots+p_{2 k}^{\prime}+\cdots+p_{2[r / 2]}^{\prime}
$$

where $p_{2 k}^{\prime}$ is the probability that $2 k \mathrm{~B}$ spins are present and form antiferromagnetic clusters of even size. Due to (C.1) and the final remark to the lemma, one has

$$
\begin{gathered}
p_{0}^{\prime}=p_{0}=x^{r} \\
p_{2 k}^{\prime} \geqslant p_{2 k} t^{2 k-1}=\left(\begin{array}{c}
r-k \\
k
\end{array}\right) x^{r-2 k}(1-x)^{2 k} t^{2 k-1}, \\
k \geqslant 1 .
\end{gathered}
$$

Therefore a lower bound of $p_{r}^{\prime}(x)$ is

$$
b_{r}(x)=x^{r}+\sum_{k=1}^{k=2[r / 2]} p_{2 k} t^{2 k-1}
$$

so that $w_{r}(x)<1-b_{r}(x)$. 
Now $b_{r}(x)$ can be evaluated by the method used for (B.3), and one finally obtains :

$$
\begin{aligned}
t\left(b_{r}(x)-x^{r}\right) & +x^{r}=\frac{x^{r}}{D(x)} \times \\
\times & {\left[\left(\frac{1+D(x)}{2}\right)^{r+1}-\left(\frac{1-D(x)}{2}\right)^{r+1}\right] }
\end{aligned}
$$

where

$$
D(x)=\left[1+4 t^{2}\left(\frac{1-x}{x}\right)^{2}\right]^{1 / 2} .
$$

This yields the desired result for large values for $r$ :

$$
b_{r}(x) \approx x^{r}\left[1+t^{2}\left(\frac{1-x}{x}\right)^{2}\right]^{r+1} \approx y^{\prime r}
$$

with

$$
y^{\prime}=x+t^{2} \frac{(1-x)^{2}}{x} \text {. }
$$

Or, to second order in $(1-x)$

$$
y^{\prime} \approx x+t^{2}(1-x)^{2} .
$$

Therefore, as explained above, a sufficient condition for $\Pi_{i j}$ to vanish at infinite distance is (returning to notation $x_{\mathrm{B}}$ for $x$ ) :

$$
x_{\mathbf{B}}+\left(1-x_{\mathbf{B}}\right)^{2} t^{2}>X\left(x_{\mathbf{A}}\right)=X,
$$

or

$$
x_{\mathrm{B}}>X-\left(\frac{1-X}{1+\exp -4 \beta\left|J_{\mathrm{BB}}\right| \cosh 8 \beta J_{\mathrm{AB}}}\right)^{2} .
$$

Condition (7.2) follows a fortiori when condition (5.1) is satisfied.

\section{References}

[1] Phani, M. K., Lebowitz, J. L., Kalos, M. H., Tsai, C. C., Phys. Rev. Lett. 42 (1979) 577.

[2] André, G., Bidaux, R., Carton, J.-P., Conte, R., de Seze, L., J. Physique 40 (1979) 479.

[3] Villain, J., J. Phys. C 10 (1977) 1717.

[4] Toulouse, G., Comm. Phys. 2 (1977) 115

[5] See for instance Steiner, M., Villain, J., Windsor, C. G., Adv. Phys. 25 (1976) 200 and Ref. therein.

[6] Paquet, D., J. Chem. Phys. 66 (1977) 886.

[7] Chang, C. H., Phys. Rev. 88 (1952) 1422.

[8] Villain, J., Z. Phys. B 33 (1979) 31.

[9] This statement may seem to rely on a particular choice of boundary conditions : if the B chains had odd number of spins the ground state would be ferromagnetic. However, a more realistic description of these boundary conditions, such as those met in a real crystal, would require inspection of what happens in the presence of irregular edges, i.e. when the length of each chain is allowed to fluctuate about a fixed mean value. In this case, any B chain connected with its neighbouring A chains via an even number of spins will amount to a zero energy interface between two parts of the system which will be uncorrelated. Nevertheless, even in presence of these irregular edges boundary conditions, long range correlation is restored as soon as temperature becomes non strictly zero as shown in section 3. In other words, with respect to the virtual set of the various physically conceivable boundary conditions, only a zero measure subset allows for an ordered state at $T=0$. 